\title{
Medication-based urolithiasis and atazanavir
}

\author{
Paul M. Koblic MD, Wayne L. Gold MD, Charles J.L. la Porte PharmD PhD, Todd C. Lee MD
}

Competing interests: Charles la Porte, in his capacity at the Ottawa Hospital Research Institute, has received research grants or payments for lectures or consultations from Abbott Laboratories, Boehringer Ingelheim, Bristol-Myers Squibb, E-MedHosting, Merck, Pfizer, Roche and Tibotec. No competing interests declared by Paul Koblic, Wayne Gold and Todd Lee.

This article has been peer reviewed.

Correspondence to: Dr. Todd C. Lee, todd.lee@utoronto.ca

CMAJ 2011. DOI:10.1503 /cmaj.100019
A 57-year-old man with chronic HIV infection reported an eight-month history of passing stones and a chalky white discharge from his urethra. He also reported dysuria, but had not experienced fever or symptoms of renal colic. His antiretroviral therapy included abacavir $600 \mathrm{mg}$ per day, lamivudine $300 \mathrm{mg}$ per day and atazanavir $300 \mathrm{mg}$ per day, boosted with ritonavir $100 \mathrm{mg}$ per day. He had been following this regimen for 11 months. His $\mathrm{CD}_{4}$ cells were $1098 / \mu \mathrm{L}$ and his viral load was undetectable $(<50$ copies $/ \mathrm{mL})$.

Four months earlier, the patient had been treated for acute renal failure that was attributed to an episode of enterococcal pyelonephritis. At that time, an abdominal ultrasound had shown mild left pelvicaliectasis with diffuse thickening of the left renal pelvis and ureter, without evidence of an obstructing stone or mass. The patient's dietary intake of calcium and meat products was normal. His father had a history of renal colic.

On examination, the patient's vital signs were within normal ranges. There was no costovertebral angle tenderness. The remainder of the examination was also normal. The results of a complete blood count and routine serum electrolyte measurements were normal. His creatinine was 84 (normal < 109) $\mu \mathrm{mol} / \mathrm{L}$. Metabolic evaluation for causes of urolithiasis showed normal serum calcium, magnesium and phosphate measurements. His serum urate concentration

\section{- Ker POINTS}

- Stone composition analysis should be part of the routine investigation of all new instances of urolithiasis.

- Because stones can be caused by specific medications, a medicationexposure history is essential in determining the cause of urolithiasis.

- Physicians should be aware of urolithiasis as a potential complication of therapy with atazanavir and should consider the role of this medication in the setting of new-onset urolithiasis.

was 503 (normal < 449) $\mu \mathrm{mol} / \mathrm{L}$ and his urinary $\mathrm{pH}$ was 6.0. A 24-hour urine collection for creatinine clearance, calcium, magnesium, phosphate and uric acid excretion were all normal. Calculi collected by the patient (Figure 1) were sent for composition analysis by a validated liquid chromatography tandem-mass spectrometry method. Nine samples were analyzed and determined to be composed of 37\%-65\% unmetabolized atazanavir by weight. Ritonavir was detected in trace amounts.

\section{Discussion}

In North America, the lifetime risk of urolithiasis is estimated at $10 \%-15 \%$, with a $50 \%$ rate of recurrence over 10 years. ${ }^{1}$ The cost of the acute management of urolithiasis in the United States is estimated at $\$ 1.83$ billion annually. ${ }^{2}$ More than $80 \%$ of instances involve calcium-based stones, with calcium oxalate stones occurring more commonly than calcium phosphate stones. Uric acid stones occur in 5\%-10\% of instances, followed in lower incidence by cystine stones and struvite stones, the latter of which are composed of magnesium-ammonium-phosphate.

A variety of renal conditions are associated with urolithiasis, including type 1 renal tubular acidosis, medullary sponge kidney, primary hyperoxaluria, and urinary tract infection with urea-splitting organisms. ${ }^{3}$ Nonrenal conditions associated with stone development include hyperparathyroidism, conditions predisposing to development of hyperuricemia, and Crohn disease, which may result in development of calcium oxalate stones.

\section{Determining the cause}

Comprehensive guidelines for diagnosis and management of patients with urolithiasis (updated in 2010) are available from the European Association of Urology website (www .uroweb.org/guidelines/online-guidelines). ${ }^{4}$ For 
all patients presenting with urolithiasis, a detailed history is indicated, with emphasis on dietary intake, family history of urolithiasis, conditions associated with development of urinary stones and a complete medication history.

Because of consideration for cost, full metabolic evaluation for causes of urinary stone formation has historically been reserved for patients with recurrent stones or patients with risk factors for further stone development, such as young age and a positive family history. Recent work, however, has shown a significant cost-benefit to early metabolic work-up and initiation of secondary prophylactic measures for renal colic when compared with repeated acute interventions. ${ }^{5}$

Patients with a single episode of renal colic and no risk factors for recurrence can be streamed into a simplified evaluation, whereas an extended work-up is warranted for patients who have recurrent formation of stones or important risk factors for recurrence. ${ }^{4,6}$ These risk factors are outlined in Box 1.6,7 For low-risk patients, investigations typically include measurements of creatinine, serum electrolytes, serum calcium, magnesium, phosphate and urate levels as well as a urinalysis, urinary $\mathrm{pH}$ and culture (Box 2)., ${ }^{4,6}$ Some experts also recommend 24-hour urine collections for quantitation. ${ }^{4}$ Stone composition analysis is recommended for all first instances of urolithiasis. ${ }^{4,6}$

Extended evaluation of patients with recurrent stones or ongoing risk factors for development of stones should include measurement of serum parathyroid hormone and vitamin D levels in patients with hypercalcemia and a 24-hour urine sample for volume, creatinine clearance, electrolytes, calcium, magnesium, phosphate, oxalate, uric acid and quantitation of citrate (Box 2), ${ }^{4,6}$

In our patient, more common metabolic causes for his urolithiasis were initially considered. When testing failed to show a cause, the stones were sent for composition analysis in consideration of a diagnosis of medication-based urolithiasis related to his antiretroviral therapy.

Although this paper focuses on determining the cause of urolithiasis rather than management of an acute episode of urinary colic, it should be noted that patients with recurrent urolithiasis may experience substantial exposure to radiation with repeated computed tomography scans in acute episodes. This issue should be taken into consideration in subsequent typical presentations to the emergency department by these patients. ${ }^{8}$

\section{Medication-based urolithiasis}

Stones caused by medications represent about $1 \%-2 \%$ of all uroliths. ${ }^{9}$ For the purposes of this discussion, the term "medication-based urolithiasis" refers to stones formed by direct crystallization of a poorly soluble, renally excreted medication or its metabolites, as well as to stones formed when medications crystallize around previously formed urinary stones. Patients who have risk factors for more common causes of urolithiasis are also at increased risk for stones caused by medications. Our use of the term "medication-based urolithiasis" is not intended to describe stones that develop in relation to the metabolic effects of medication therapy, such as calcium phosphate stones resulting from the exogenous administration of vitamin D or calcium. On computed tomographic scans, medication-based stones may not differ in appearance from other subtypes of stones, and consequently, can be confirmed only with stone analysis.

Sulfonamides were the first medications linked to urolithiasis. ${ }^{8}$ They have since been joined by a variety of other antibiotics, antihypertensive drugs, antacids and other medications,

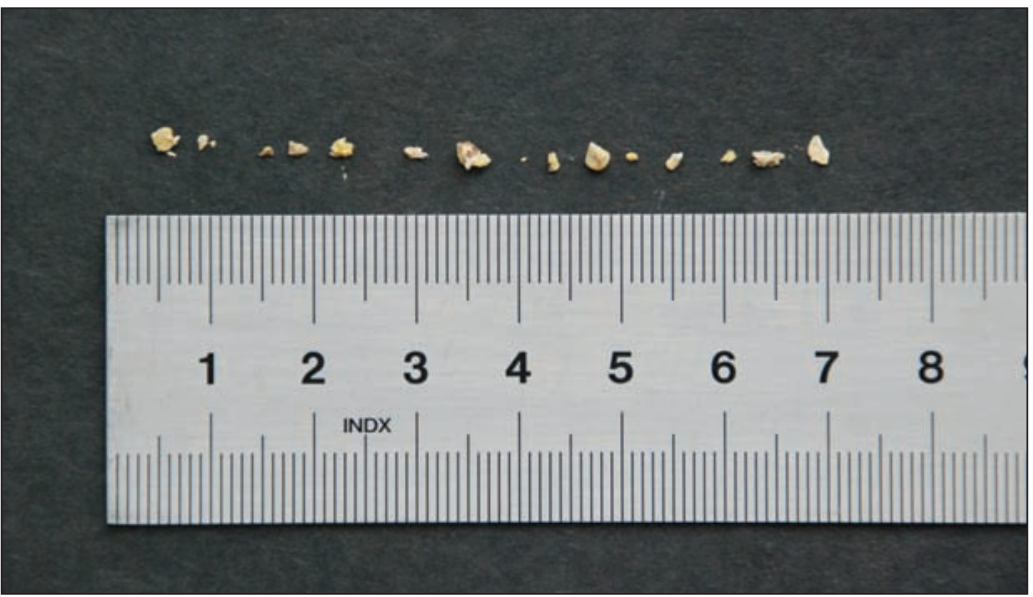

Figure 1: Urinary calculi passed by a $57-$ year-old man who had been on antiretroviral therapy for 11 months.
Box 1: Factors indicating high risk for recurrent urolithiasis ${ }^{6,7,15}$

- Family history of urolithiasis

- Obesity

- Residual stone fragments or bilateral, vast stone burden

- Disorders predisposing to hyperuricemia, including diabetes mellitus

- Hyperparathyroidism

- Cystinuria

- Primary hyperoxaluria

- Type 1 renal tubular acidosis

- Medullary sponge kidney

- Gastrointestinal malabsorption syndromes, including Crohn disease 
including the HIV-1 protease inhibitors indinavir and nelfinavir (Table 1). ${ }^{9,10}$ Interestingly, although indinavir-based urolithiasis is common (occurring in $12.4 \%$ of patients in some series), ${ }^{11}$ our patient had never developed renal calculi despite 10 years of prior therapy with that agent. Indinavir had been discontinued 16 months before his presentation with renal stones because the patient had developed a rash.

In 2006, atazanavir became the latest antiretroviral agent with a reported link to urolithiasis. ${ }^{12}$ Atazanavir is an azapeptide inhibitor of HIV-1 protease; since the 2008 update of guidelines for the treatment of HIV infection, it has been included as a first-line choice of protease inhibitor in highly active antiretroviral therapy. ${ }^{13}$ Atazanavir is metabolized by the cytochrome P450 3A4 isoenzyme in the liver, and about $13 \%$ of each dose is excreted in the urine, with $7 \%$ excreted unmetabolized. ${ }^{3}$ Atazanavir becomes less soluble in alkaline urine, yet the exact mechanism of stone formation is currently unknown. The urinary $\mathrm{pH}$ of our patient was 6.0, which may have contributed to the formation of stones, given that atazanavir is maximally soluble at a urinary $\mathrm{pH}$ of 1.9 .

Since 2002, the US Adverse Event Reporting System has collected details on 30 instances of urolithiasis in patients treated with atazanavir. ${ }^{14}$ Among these patients, the median time from initiation of therapy with atazanavir to the first documented renal stone was 1.7 years. Our patient developed urolithiasis 11 months after initiating therapy with atazanavir. Of 14 reported instances for which stone composition analysis was per-

\section{Box 2: Recommended routine laboratory} investigation of urolithiasis ${ }^{4,6}$

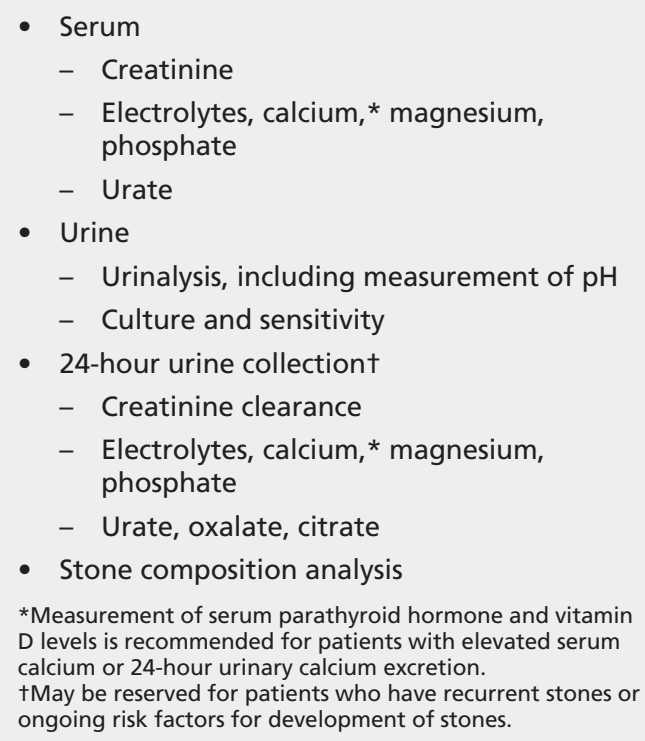

* Measurement of serum parathyroid hormone and vitamin $D$ levels is recommended for patients with elevated serum calcium or 24-hour urinary calcium excretion.

tMay be reserved for patients who have recurrent stones or ongoing risk factors for development of stones.

formed, 12 patients had stones that were positive for atazanavir, varying between $40 \%$ and $100 \%$ composition by weight.

\section{Prevention}

Preventive regimens should be tailored to the specific causes that are detected. ${ }^{4,6}$ In general, fluid intake should be increased to at least $2 \mathrm{~L}$ of water per day in patients without contraindications such as congestive heart failure or cirrhosis. There is good evidence from randomized trials that dietary modifications, including salt restriction, may also reduce recurrent stone formation. ${ }^{4}$ Additional dietary modifications should be based on any biochemical abnormalities that are identified in the investigation of the cause of stone development. ${ }^{4}$ In patients with low 24-hour excretion of urinary citrate, prophylaxis with potassium citrate may be considered.

When appropriate alternatives exist, other medications should be substituted in patients with medication-based urolithiasis. This strategy has been used in the management of indinavirbased stones with variable success. ${ }^{11}$

Our patient's protease inhibitor was changed from atazanavir to lopinavir. He was also instructed to increase his daily fluid intake to at least $2 \mathrm{~L}$ per day. His passage of stones promptly ceased. Imaging of the abdomen performed nine months later showed normal-sized kidneys and no hydronephrosis. The previously noted uroepithelial thickening and mild left pelvicaliectasis had completely resolved. In retrospect, those findings may have been evidence of the crystallization of atazanavir in the renal collecting system at the time of the patient's presentation with enterococcal pyelonephritis.

Table 1: Commonly used medications implicated in the development of urolithiasis ${ }^{8,9}$

\begin{tabular}{|c|c|}
\hline Class of medication & Example(s) \\
\hline \multicolumn{2}{|l|}{ Antibiotics } \\
\hline Aminopenicillins & Ampicillin, amoxicillin \\
\hline Cephalosporins & Ceftriaxone \\
\hline Furanes & Nitrofurantoin \\
\hline Quinolones & Norfloxacin, ciprofloxacin \\
\hline Sulfonamides & $\begin{array}{l}\text { Sulfamethoxazole, } \\
\text { sulfadiazine }\end{array}$ \\
\hline Antihypertensives & Triamterene \\
\hline Antivirals & Acyclovir \\
\hline $\begin{array}{l}\text { HIV-1 protease } \\
\text { inhibitors }\end{array}$ & $\begin{array}{l}\text { Indinavir, nelfinavir, } \\
\text { atazanavir }\end{array}$ \\
\hline $\begin{array}{l}\text { Xanthine oxidase } \\
\text { inhibitors }\end{array}$ & Allopurinol \\
\hline
\end{tabular}




\section{Conclusion}

Stone composition analysis should be a routine part of the evaluation of patients with newonset urolithiasis. In our patient's instance, this analysis led to the determination that his stones were composed of atazanavir, resulting in its substitution with another protease inhibitor. Given that the guidelines for antiretroviral therapy of adult HIV infection now recommend atazanavir as a first-line choice of protease inhibitor, treating physicians should be aware of this potential complication of therapy and consider its role in the setting of new-onset urolithiasis.

\section{References}

1. Teichman JMH. Acute renal colic from ureteral calculus. $N$ Engl J Med 2004;350:684-93.

2. Trinchieri A. Epidemiological trends in urolithiasis: impact on our health care systems. Urol Res 2006;34:151-6.

3. Moe OW. Kidney stones: pathophysiology and medical management. Lancet 2006;367:333-44.

4. Turk C, Knoll T, Petrik A, et al. Guidelines on urolithiasis. Arnhem (The Netherlands): European Association of Urology; 2010. Available: www.uroweb.org/gls/pdf/Urolithiasis\%202010 .pdf (accessed 2010 Oct. 4)

5. Robertson WG. Is prevention of stone recurrence financially worthwhile? Urol Res 2006;34:157-61.
6. Hesse A, Straub M. Rational evaluation of urinary stone disease. Urol Res 2006;34:126-30.

7. Reyataz. Prescription information. Princeton (NJ): BristolMyers Squibb; 2008.

8. Brenner DJ, Hall E. Computed tomography - an increasing source of radiation exposure. N Engl J Med 2007;357:2277-84.

9. Lehr D. Clinical toxicity of sulfonamides. Ann N Y Acad Sci 1957; 69:417-47.

10. Daudon M, Jungers P. Drug-induced renal calculi: epidemiology, prevention and management. Drugs 2004;64:245-75.

11. Crixivan. Prescription information. Whitehouse Station (NJ): Merck \& Co.; 2008.

12. Chang HR, Pella PM. Atazanavir urolithiasis. N Engl J Med 2006; 355:2158-9.

13. Hammer SM, Eron JJ, Reiss P, et al. Antiretroviral therapy of adult HIV infection: 2008 recommendations of the International AIDS Society — USA Panel. JAMA 2008;300:555-70.

14. Chan-Tack KM, Truffa MM, Struble KA, et al. Atazanavirassociated urolithiasis: cases from the US Food and Drug Administration's Adverse Event Reporting System. AIDS 2007; 21:1215-8.

15. Daudon $\mathrm{M}$, Traxer $\mathrm{O}$, Conort $\mathrm{P}$, et al. Type 2 diabetes increases the risk for uric acid stones. J Am Soc Nephrol 2006;17:2026-33.

Affiliations: From the Department of Medicine (Koblic, Gold, Lee); the Division of Infectious Diseases, University Health Network (Koblic, Lee), University of Toronto, Toronto, Ont.; and the University of Ottawa at the Ottawa Hospital and the Ottawa Health Research Institute (la Porte), Ottawa, Ont.

Contributors: All of the authors contributed to the preparation of this manuscript and approved the final version submitted for publication.

Acknowledgement: The authors thank Guijun Zhang for his help in conducting the stone analysis. 